\title{
Estimation of Measurement Uncertainty of kinematic TLS Observation Process by means of Monte-Carlo Methods
}

\author{
Hamza Alkhatib $^{1, *}$ and Hansjörg Kutterer ${ }^{2}$ \\ ${ }^{1}$ Geodetic Institute, Engineering Geodesy and Geodetic \\ Data Analysis Section, Gottfried Wilhelm Leibniz Uni- \\ versität Hannover, Germany \\ ${ }^{2}$ Bundesamt für Kartographie und Geodäsie, Frankfurt am \\ Main
}

\begin{abstract}
In many cases, the uncertainty of output quantities may be computed by assuming that the distribution represented by the result of measurement and its associated standard uncertainty is a Gaussian. This assumption may be unjustified and the uncertainty of the output quantities determined in this way may be incorrect. One tool to deal with different distribution functions of the input parameters and the resulting mixed-distribution of the output quantities is given through the Monte Carlo techniques. The resulting empirical distribution can be used to approximate the theoretical distribution of the output quantities. All required moments of different orders can then be numerically determined. To evaluate the procedure of derivation and evaluation of output parameter uncertainties outlined in this paper, a case study of kinematic terrestrial laserscanning (k-TLS) will be discussed. This study deals with two main topics: the refined simulation of different configurations by taking different input parameters with diverse probability functions for the uncertainty model into account, and the statistical analysis of the real data in order to improve the physical observation models in case of k-TLS. The solution of both problems is essential for the highly sensitive and physically meaningful application of k-TLS techniques for monitoring of, e. g., large structures such as bridges.
\end{abstract}

Keywords. Monte Carlo simulation, kinematic terrestrial laserscanning, uncertainty modeling.

\section{Introduction}

The main tasks of an engineer include design, produce, and test of structures devices, and processes. These tasks

Corresponding author: Hamza Alkhatib, Geodetic Institute, Engineering Geodesy and Geodetic Data Analysis Section, Gottfried Wilhelm Leibniz Universität Hannover, Germany.

E-mail: alkhatib@gih.uni-hannover.de.

Received: September 18, 2012. Accepted: January 30, 2013. will be involved with mathematical and physical modeling of the different phenomena. In the constructed mathematical/physical model some information about constants parameters and functional variables are needed. Uncertainties evolve into his modeling together with these information. The sources of uncertainties result from the data or the measurements, from the statistical evaluation of the model and from the physical model.

The Guide to the Expression of Uncertainty in Measurement (GUM) is the standard reference in uncertainty modeling in engineering and mathematical science, cf. [6]. GUM groups the occurring uncertain quantities into Type A and Type B. Uncertainties of Type A are determined with the classical statistical methods, while Type B is subject to other uncertainties like knowledge about an instrument. Whereas the uncertainties of Type A can be estimated based on the measurement itself, the estimated uncertainties of Type B are based on expert knowledge, e.g., the technical knowledge about an instrumental error source.

The extension of GUM [7] recommends the propagation of uncertainties using a probabilistic approach. Within the mentioned approach the propagation of uncertainties is numerically treated by Monte Carlo (MC) techniques. The difference between the GUM [6] and the extension of GUM [7] in case of nonlinearity and/or non-Gaussianity will not significantly differ in the first and the second central moments but rather in the estimate of the confidence region, which is reflected in the non-Gaussian PDF of the output quantities. The acceptance of MC techniques has significantly increased during the last decade. Consequently, it's widely used within many scientific disciplines. [5] suggested to use MC simulations instead of the treatment of the combined uncertainties by applying the law of error propagation (LOP). [15] recommended a MC based method to evaluate the measurement uncertainties in nonlinear models. [9] suggested the determination of the uncertainty according to GUM by a Bayesian confidence interval using MC simulation. The approach has been explained in detail and applied to the results of terrestrial laserscanning (TLS). Furthermore, the approach has been extended in [10] to evaluate uncertainties of correlated measurements by another application in TLS. In [11] the 2D case of kinematic TLS was studied where repeated profile scans are observed from a fixed station with a high repetition frequency for monitoring purposes. The focus was put on a refined modeling of the uncertainty of both the observations and the derived positions of the profile points. MC simula- 
tion techniques were applied to provide numerical results for discussion and validation.

The paper is organized as follows: First we will describe the general idea of $\mathrm{MC}$ techniques to describe measurement uncertainties in the context of GUM. The application example to kinematic TLS is given and discussed in the following sections.

\section{UNCERTAINTY MODELING WITH MONTE CARLO TECHNIQUES}

In MC techniques, both, the random and the systematic components of the uncertainty, are treated as having a random nature. Please note that not the systematic component itself is modelled as random, it is the knowledge about the systematic component for which a probability distribution is introduced [8].

The GUM suggests in some cases to select the probability distribution function (PDF) of the input quantities as rectangular, triangular, and trapezoidal [6]. In these cases, it is hard/impossible to obtain the estimate of the uncertainty for the output quantity in a closed mathematical form. An alternative to modeling and propagating uncertainties is propagating distributions by MC simulations of the observation model from (1):

$$
y=f\left(p_{1}, p_{2}, \ldots p_{n}\right)=f(\mathbf{p}) .
$$

Here $y$ represents a random output quantity and $p_{1}, p_{2}, \ldots, p_{n}$ are the $n$ random inputs.

\subsection{Approach to Evaluate Uncertainty}

The MC techniques are of great importance for uncertainty evaluation. With a set of generated samples the distribution function for the value of the output quantity $y$ in (1) will be numerically approximated. In general, the functional relations between the basic influence parameters, referring to (1), the observations and the parameters of interest are nonlinear, and the normal distribution is not the adequate probability density function. In such case, MC simulation is a suitable way to approximately derive the stochastic properties of the quantities of interest (output quantities). It is assumed that the functional model is completely formulated relating the output quantities with the input quantities: the observations and the basic influence parameters, respectively. It is further assumed that the probability densities of the considered input quantities are a priori known. Then, a sample vector of the input quantities can be drawn repeatedly using random number generators. Random numbers are generated on a computer by means of deterministic procedures. In particular, rectangular distributed random numbers are generated, which may then in turn be transformed into random numbers of random variables having other distributions, for instance, into numbers of a normally distributed random variable [3].

For each input sample vector the corresponding values of the output quantities are calculated by using the corresponding functional relation. The set of output sample vectors yields an empirical distribution which can be used to approximate the correct random distribution of the output quantities. All required measures (expectation values, variances and covariances) as well as higher order central moments such as skewness and kurtosis can then be derived. To sum up, MC approaches to estimate the uncertainty include the following steps:

- Step 1: A set of random samples, which have the size $n$, is generated from the PDF for each random input quantity $p_{1}, p_{2}, \ldots, p_{n}$. The sampling procedure is repeated $M$ times for every input quantity.

- Step 2: The output quantities $y$ be will then calculated by:

$$
y^{(i)}=f\left(p_{1}^{(i)}, p_{2}^{(i)}, \ldots p_{n}^{(i)}\right)=f\left(\mathbf{p}^{(i)}\right),
$$

where $i=1, \ldots, M$ are the generated samples of the random input quantity $p$.

- Step 3: Particularly relevant estimates of any statistical quantities can be calculated as the expected value of the output quantity:

$$
\hat{E}(f(\mathbf{p}))=\hat{E}(y)=\frac{1}{M} \sum_{i=1}^{M} f\left(\mathbf{p}^{(i)}\right),
$$

the estimate of the variance of the output quantity [1]:

$$
\begin{aligned}
\hat{\mathbf{D}}(y)= & \frac{1}{M} \sum_{i=1}^{M}\left(f\left(\mathbf{p}^{(i)}\right)-\hat{E}(f(\mathbf{p})) \ldots\right. \\
& \left(f\left(\mathbf{p}^{(i)}\right)-\hat{E}(f(\mathbf{p}))^{T} .\right.
\end{aligned}
$$

and the skewness of univariate output quantities $y^{(1)}, y^{(2)}, \ldots, y^{(M)}$

$$
\hat{s k}(y)=\frac{1}{(M-1) \hat{s}^{3}} \sum_{i=1}^{M}\left(f\left(\mathbf{p}^{(i)}\right)-\hat{E}(f(\mathbf{p}))^{3}\right.
$$

where $\hat{E}(f(\mathbf{p}))$ is the mean value of the output quantities (see (3)), $s$ is its standard deviation $s=\sqrt{\hat{\mathbf{D}}(y)}$ (refer to (5) and (6)), and $M$ is the number of generated samples. The skewness for a normal distribution is zero, and any symmetric data should have a skewness near zero, see, e. g., [12].

For univariate output quantities the kurtosis can be 
estimated by

$$
\hat{k u}(y)=\frac{1}{(M-1) \hat{s}^{4}} \sum_{i=1}^{M}\left(f\left(\mathbf{p}^{(i)}\right)-\hat{E}(f(\mathbf{p}))^{4} .\right.
$$

The kurtosis for a standard normal distribution is 3 .

Figure 1 shows a diagram with the main steps of uncertainty modeling with a different treatment of the random and systematic uncertainties.

In [9] and [10] the above mentioned MC algorithm in case of TLS uncertainty assessment have been discussed. [2] apply it to k-TLS vertical profile scans and they combine it with a deterministic approach based on fuzzy sets. Here, only MC techniques will be considered but it is extended to the discussion of the properties of the derived time series and of their validation using real $\mathrm{k}$-TLS observation data.

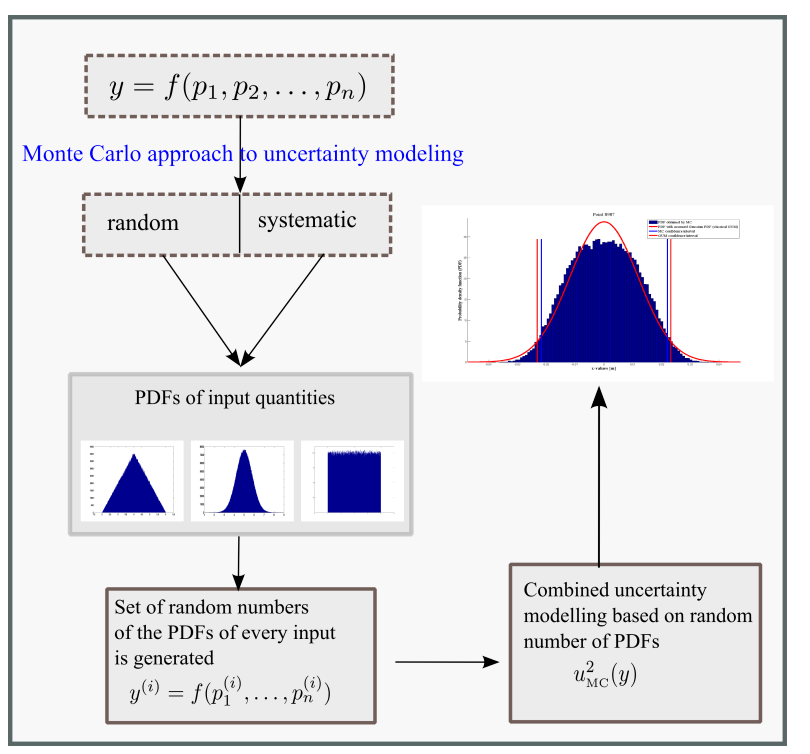

Figure 1. Treatment of uncertainty components in MC approach.

\section{APPLICATION OF THE MONTE CARLO- APPROACH TO K-TLS}

\subsection{Object and Setup}

In this section a short numerical example for the approach, presented in Section 2, is shown. The aim of the application is to detect the vertical displacements of a bridge under load, e.g., due to car traffic or train crossings. For this reason, a laserscanner of type Zoller+Fröhlich Imager 5006 scanner was placed beneath the bridge which is located in the southern part of Germany. [11] give a detailed description of the bridge, of the loading tests with different trucks, of the applied observation procedures and of the derived

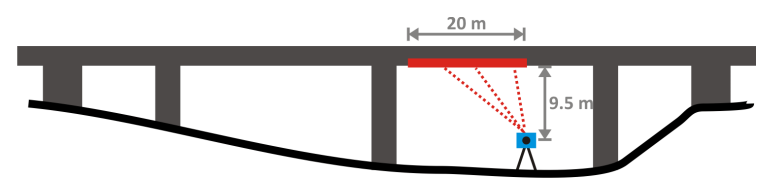

Figure 2. Bridge and scanner.

data; see Figure 3 for a graphical representation of the object and the location of the laser scanner. The horizontal section in along-track direction of the bridge ( $y$-axis) considered here has a length of $20 \mathrm{~m}$ with a shortest distance between scanner and bridge of about $9.5 \mathrm{~m}$. [16] show new analysis results of the k-TLS observations. Moreover, they compare these results with strain gauge observations and with numerical simulations based on finite-element models. Note that the consistency of all three kinds of data is better than $1 \mathrm{~mm}$. Here, the unloaded state of the Autobahn bridge is studied which was repeatedly observed in order to get a reference geometry for the analysis of the load-induced deformations. For the observation of the vertical profiles a repetition rate of 12.5 profiles per second was used while the repetition frequency of the distance measurements was $500 \mathrm{kHz}$. For the vertical angle this yields an increment of 10 mgon. There are 7216 points per epoch within the observed section; 500 profiles representing the unloaded state were considered in total.

The profile data were processed according to the procedure outlined in Section 2. In order to prepare a meaningful model for the MC simulations the observed and processed data were roughly checked regarding the level of variance and with respect to possible quantities of influence. Within this analysis also an effect in the original data was detected which seems to be due to a different temporal resolution of distance and vertical angle observation; it is indicated in Figure 3. This effect has been modeled accordingly for the simulations.

\subsection{SIMULATION OF K-TLS PROFILES}

The functional model, which was used in [2], has been established for the simulations. The time series of the vertical height $z$ of every point of the bridge can be expressed in the local coordinate system of the laserscanner by the following equation:

$$
z=d \cdot \cos (\zeta), \zeta=\zeta_{0}+\Delta \zeta
$$

with $d$ the observed distance between laser scanner and object point which induces a constant and a distanceproportional effect, $\zeta_{0}$ the observed zenith angle with a constant angular effect, and $\Delta \zeta$ the discretization term which is induced by the angular increment of the vertical servomotor. In this study seven uncertainty components were modeled: 

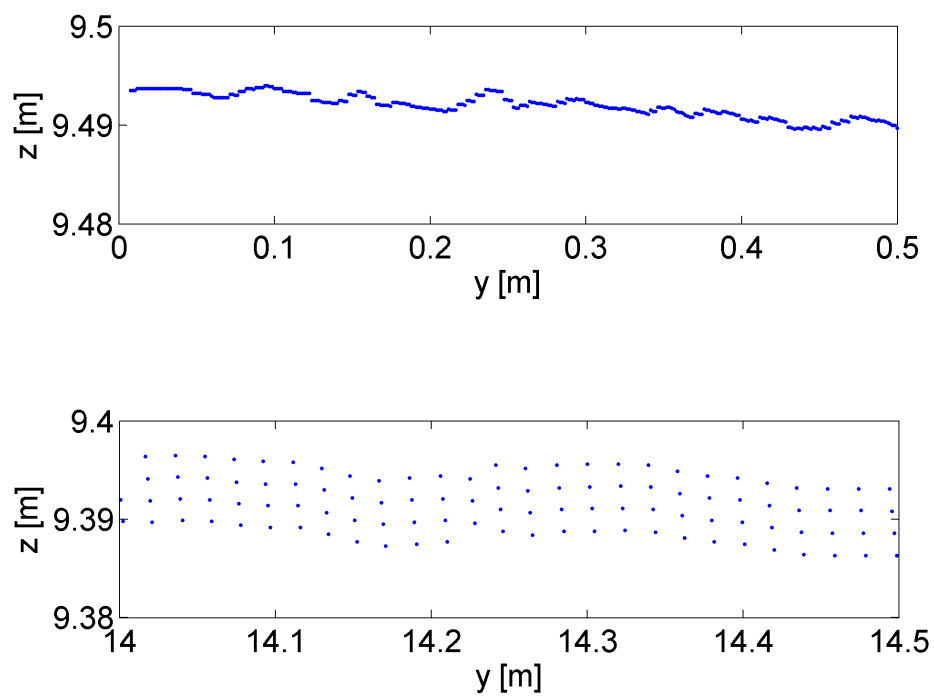

Figure 3. Two zooms into a representative profile scan $-y$-coordinate (along bridge) and z-coordinate (height with inflated scale); upper figure: section directly above the laser scanner in a spatial distance of about $9.5 \mathrm{~m}$ with orthogonal angle of incidence; lower figure: section in a spatial distance of about $17 \mathrm{~m}$ with oblique angle of incidence.

- Uncertainty of the distance ( $p_{1}$, Type A), and their additional constant $\left(p_{2}\right.$, Type B),

- distance depending term for the uncertainty of the distance measurement $\left(p_{3}\right.$, Type B, see [9]),

- incidence angle of the measured distance under the bridge $\left(p_{4}\right.$, Type B),

- uncertainty of the zenith angle ( $p_{5}$, Type A) and the vertical index error $\left(p_{6}\right.$, Type B),

- vertical resolution for the zenith angle (the step width of the motor) ( $p_{7}$, Type B).

The uncertainties and the PDF for the input quantities $p_{i}, i \in\{1, \ldots, 7\}$ are given in Table 1 .

The symbols $\mu$ and $\sigma^{2}$ in Table 1 denote the expectation value and the variance of the random variable, respectively; the uniform and the triangular distribution are defined by the lower bound $p_{i l}$ and the upper bound $p_{i u}$ of the interval with positive values of the density function. The assumptions for the uncertainties of $p_{1}, p_{5}$ and $p_{6}$ are based on the technical data from the manufacturer and for the uncertainties of $p_{2}, p_{3}$ and $p_{4}$ on [14] and for $p_{7}$ on [13].

In the following, the results of three different MC simulation runs are shown and discussed which were calculated for the bridge section described in Section 3.1. In all simulations 100,000 samples were drawn for each random quantity; the obtained values were processed according to the model described in equation (1). 100,000 $\mathrm{MC}$ runs are generated as necessary to obtain the estimates of the statistical moments of the PDF with at least three significant digits.
The same results were numerically proven in [9]. Three different class widths were selected for the simulations: one / five / ten observation values per class and epoch. As representative value for each class and epoch the respective arithmetic mean of the single class values was used; this is reasonable because of the yet small class widths. Thus, only a minor spatial filtering was applied but not a temporal filter. The temporal sequences of these representative class values define the time series or data series, respectively, which are analyzed further.

Due to the unloaded state of the bridge all profiles can be considered as stationary. Therefore three central moments of the underlying probability density functions are determined empirically: standard deviation (of the single value), skewness and kurtosis. Note that expectation value and standard deviation are necessary and sufficient in order to uniquely define a normal distribution. The skewness of a normally distributed random variable equals 0 , and the kurtosis equals 3 (In order to refer the kurtosis of an arbitrary density to the normal distribution the value 3 can be subtracted; then the kurtosis of the normal distribution equals 0 ). Hence, skewness and kurtosis are well-suited to detect violations of the normal distribution assumption.

Simulation I: For this simulation, the three input quantities $\left(p_{1}, p_{3}\right.$ and $\left.p_{5}\right)$ were considered for uncertainty modeling: the constant and distance-proportional effect of the distance observation, and the constant angular effect of the zenith angle observation. The input quantities for Simulation I are defined in the left three columns of Table 2. The 


\begin{tabular}{llll}
\hline$p_{i}$ & Er. Com. & PDF & PDF Type \\
\hline$p_{1}$ & random & $p_{1} \sim N\left(\mu_{p_{1}}, \sigma_{p_{1}}^{2}\right)$ & normal \\
$p_{2}$ & systematic & $p_{2} \sim T\left(p_{2 l}, p_{2 u}\right)$ & triangular \\
$p_{3}$ & random & $p_{3} \sim N\left(\mu_{p_{3}}, \sigma_{p_{3}}^{2}\right)$ & normal \\
$p_{4}$ & random & $p_{4} \sim N\left(\mu_{p_{4}}, \sigma_{p_{4}}^{2}\right)$ & normal \\
$p_{5}$ & random & $p_{5} \sim N\left(\mu_{p_{5}}, \sigma_{p_{5}}^{2}\right)$ & normal \\
$p_{6}$ & systematic & $p_{6} \sim T\left(p_{6 l}, p_{6 u}\right)$ & triangular \\
$p_{7}$ & systematic & $p_{7} \sim U\left(p_{7 l}, p_{7 u}\right)$ & uniform \\
\hline
\end{tabular}

Table 1. Uncertainties for the input quantities.

three central moments of the empirical distributions of the respective representative class values obtained as results of the Simulation I are presented in Figure 4.

Simulation II: For the second simulation the same input quantities were used as in the first simulation; in addition, the uncertainty induced by the angular increment of the vertical servo-motor $\left(p_{7}\right)$ was modeled. The three central moments of the empirical distributions derived as results of the Simulation I are presented in Figure 5.

Simulation III: For the last simulation all input quantities described in Table 1 were used; the result is shown in Figure 6.

Looking at the standard deviations shown in Figure 4 and Figure 5, the distance-proportional effect on the standard deviations of the representative profile points is obvious. Moreover, the square-root law $\sigma_{\bar{x}} / \sqrt{n}$ for the standard deviation of the mean value $\bar{x}$ with respect to the standard deviation of the single values by the number $n$ of sample values can clearly be seen. In addition, the skewness is insignificant in both simulations. The difference lies in the kurtosis. Whereas in Figure 4 the normal distribution assumption seems to hold, it is clearly violated in Figure 5. The assumption of a Gaussian distribution in Figure 6 is not obvious. Therefore, the rigorous mathematical assessment of the discussion about this assumption has to be referred to a suitable hypothesis test. For this purpose the Kolmogorov-SmirnovTest (KS-Test) is used. The KS-Test is a form of minimum distance estimation used to compare a data set with a reference probability distribution. The test quantifies a distance between the empirical distribution function of the data set and the cumulative distribution function of the reference distribution. By modifying the KS-Test it can serve as a goodness of fit test, see, e. g. [4]. In the case of testing for normality of the distribution, the samples are standardized and compared with a standard normal distribution. As a result of the performed hypothesis, we were able to approve that only in Simulation I the normal distribution holds.

Due to the convolution different probability distributions (normal, triangular and uniform, respectively) the resulting distributions of Simulation II and III are not normal distribution, this can be validated by means of the KS-Test. Moreover (especially for Simulation II), the kurtosis values decrease from 3 (which is valid for observations directly in vertical direction and which does not contradict to the normal distribution assumption) to about 2 in a horizontal distance of about $20 \mathrm{~m}$. There are two effects which superpose each other: one from the uniform distribution and the other from the (non-linear) cosine function. In case of increasing the class width, the effect on the kurtosis is significantly mitigated possibly due to the central limit theorem of probability theory.

\section{VALIDATION OF THE SIMULATION RESULTS}

In order to assess and to validate the simulation results, actually observed profile data were processed and analyzed as well in full accordance with the procedure applied for the two simulation runs. Figure 7 shows the obtained results; like in Section 3 the standard deviations, the skewness and the kurtosis of the individual classes of height coordinates are given. The standard deviations show again a clear dependence on the horizontal distance between the scanner and the profile points; this dependence is reduced when the class width is increased. However, in contrast to the simulated data, the mentioned square-root law does not fully apply - neither for small values of the $y$-coordinate nor for large values. For small values the reduction of the variance induced by averaging is smaller than expected, for large value the reduction effect is larger than expected. Like in the simulations, the skewness of the empirical distributions of the individual classes does not significantly differ from 0 ; note that the visible variability of the values decreases when $y$ increases. Hence, the empirical distributions are symmetric - independent of the class width. However, the decrease of the kurtosis with respect to increasing values of $y$ is remarkable. On the one hand, there is a systematic and significant decrease of the values from 3 (what is expected in case of normal distribution) to a value slightly below 2 . This indicates clearly the violation of the normal distribution assumption. On the other hand however, this effect is 


\begin{tabular}{cll}
\hline & & Simulation I: without vertical increment \\
\hline Input quantity & PDF & Num. value (std. dev) \\
\hline$p_{1}$ & Normal & $0.5 \mathrm{~mm}$ \\
$p_{3}$ & Normal & $30 \mathrm{ppm}$ \\
$p_{5}$ & Normal & $10 \mathrm{mgon}$ \\
\hline & & Simulation II: with vertical increment \\
\hline Input quantity & PDF & Num. value (std. dev) \\
\hline$p_{1}$ & Normal & $0.3 \mathrm{~mm}$ \\
$p_{3}$ & Normal & $30 \mathrm{ppm}$ \\
$p_{5}$ & Normal & $5 \mathrm{mgon}$ \\
$p_{7}$ & Uniform & $20 \mathrm{mgon}$ \\
\hline & & Simulation III: with all input quantities \\
\hline Input quantity & PDF & Num. value (std. dev) \\
\hline$p_{1}$ & Normal & $0.5 \mathrm{~mm}$ \\
$p_{2}$ & Triangular & $0.4 \mathrm{~mm}$ \\
$p_{3}$ & Normal & $30 \mathrm{ppm}$ \\
$p_{4}$ & Normal & $1 \mathrm{~mm}$ \\
$p_{5}$ & Normal & $10 \mathrm{mgon}$ \\
$p_{6}$ & Triangular & $8 \mathrm{mgon}$ \\
$p_{7}$ & Uniform & $10 \mathrm{mgon}$ \\
\hline
\end{tabular}

Table 2. MC simulation: input quantities for the uncertainty models (type of probability densities and numerical values of the standard deviations).
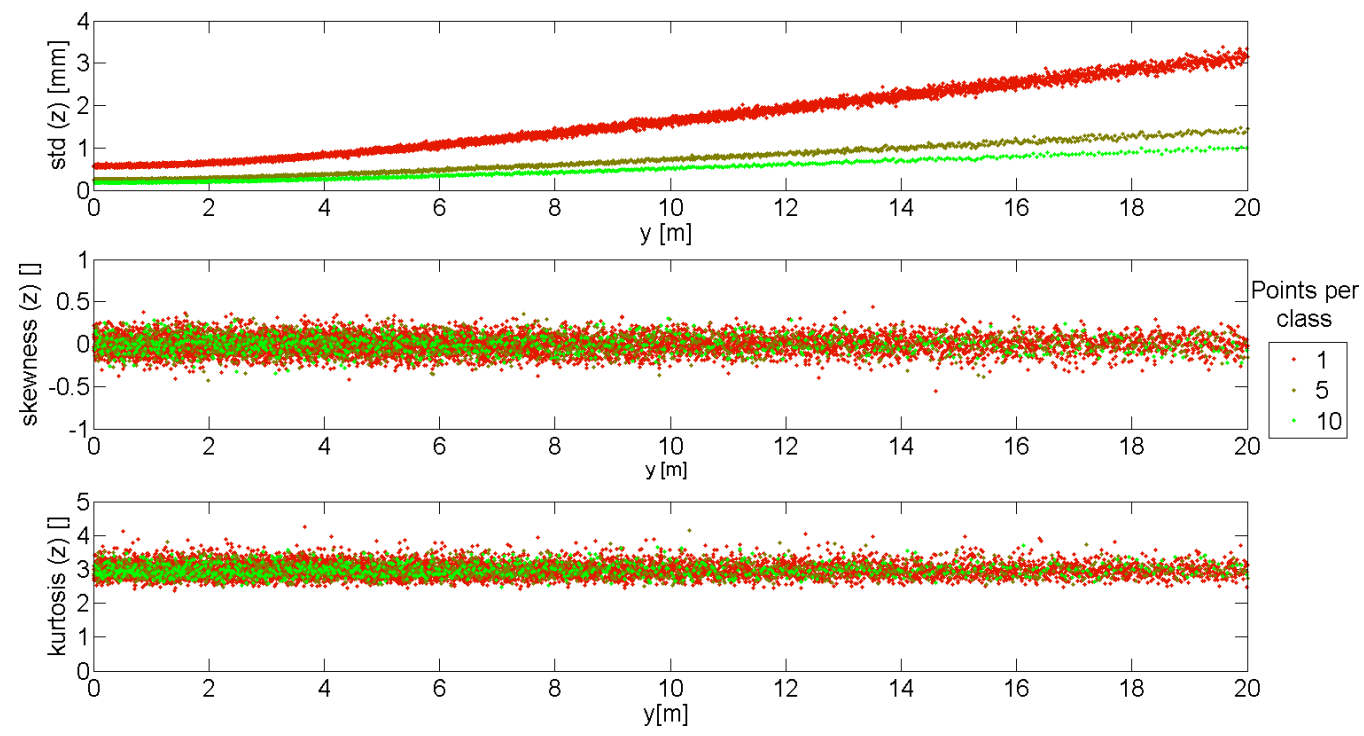

Figure 4. Simulation I - without vertical motor increment uncertainty: analysis of the simulated k-TLS profiles for three different class widths: standard deviations, skewness, and kurtosis. 

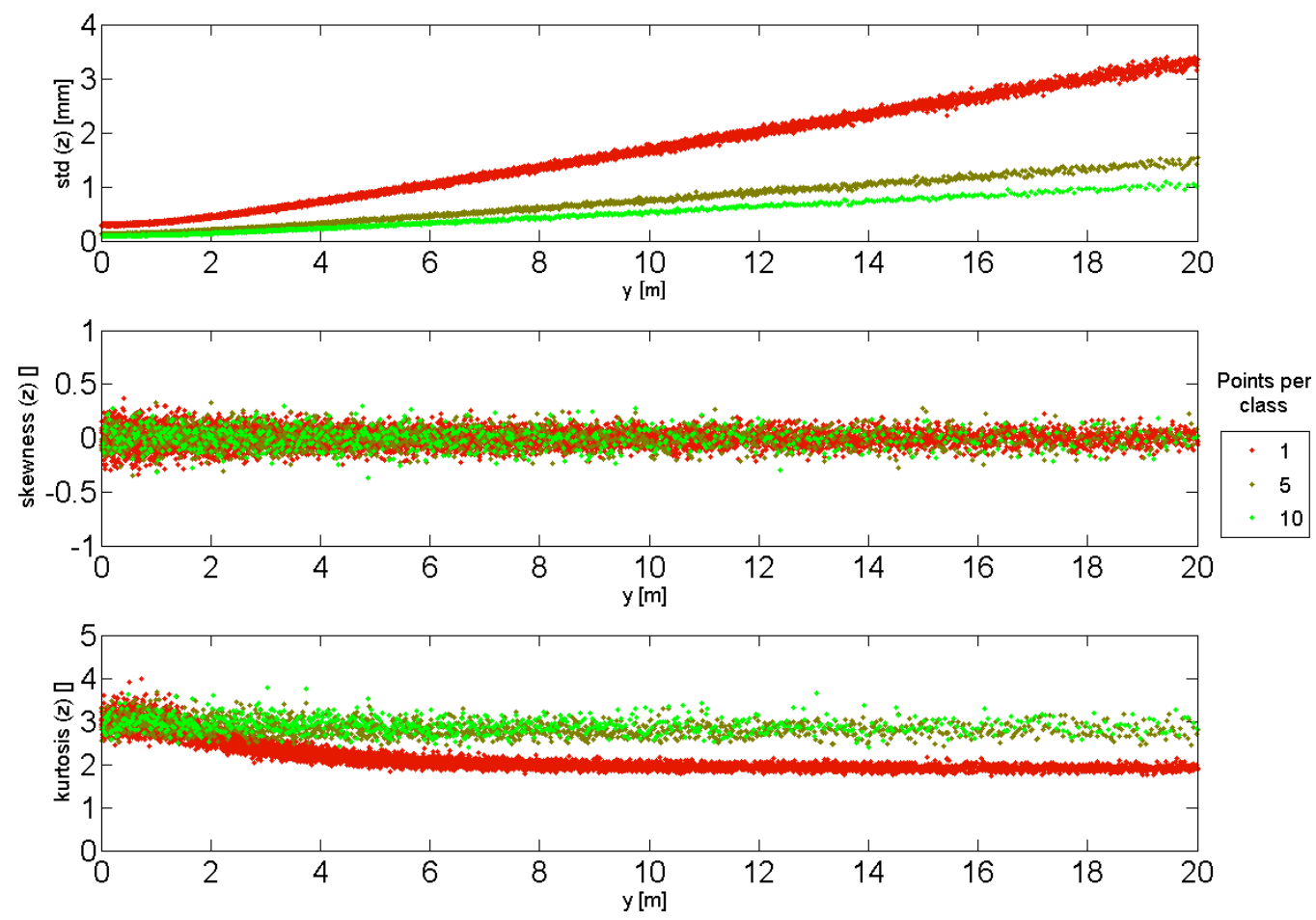

Figure 5. Simulation II - with vertical motor increment uncertainty: analysis of the simulated k-TLS profiles for three different class widths: standard deviations, skewness, and kurtosis.
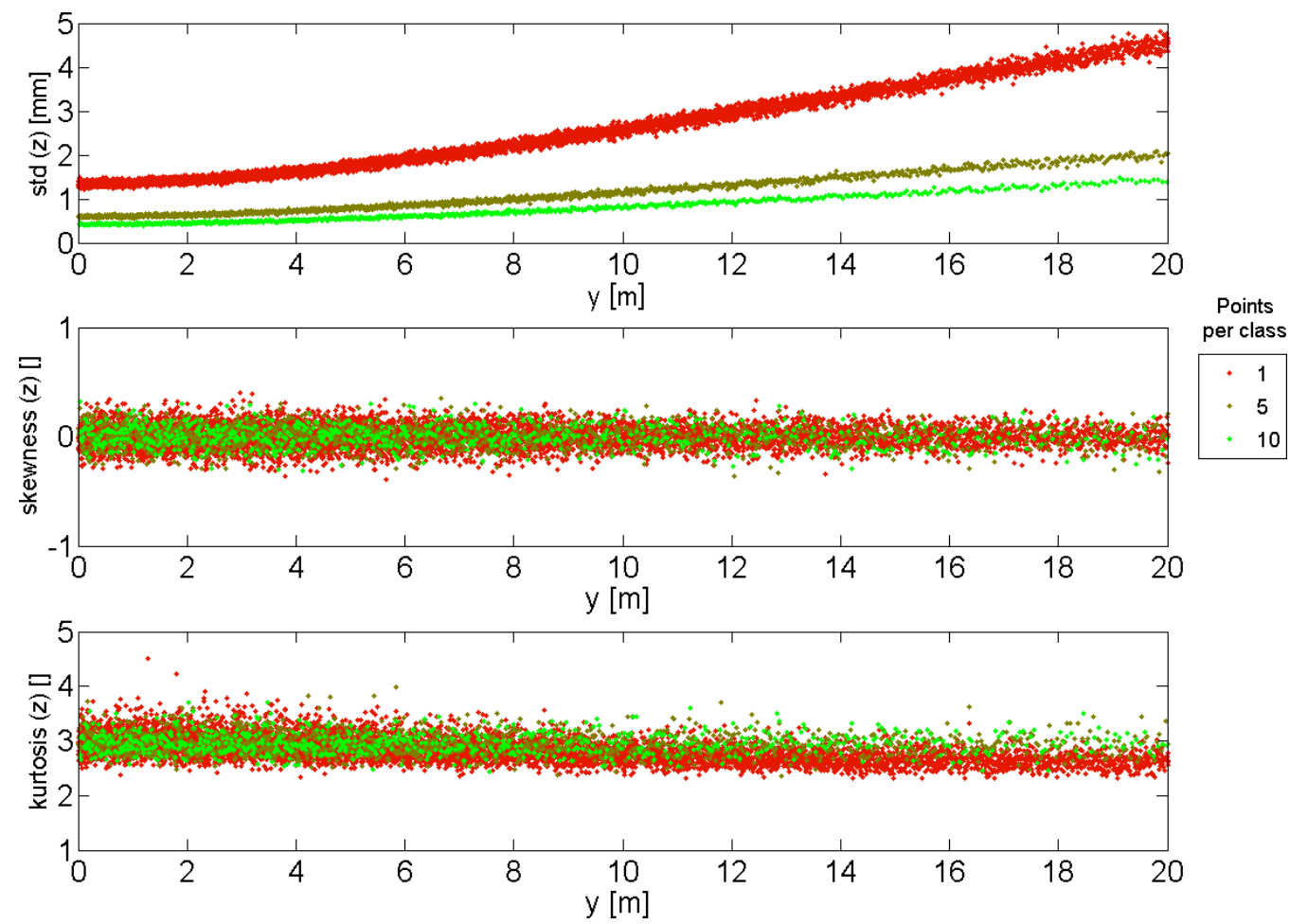

Figure 6. Simulation III - with all input quantities listed in Tab. 1: analysis of the simulated k-TLS profiles for three different class widths: standard deviations, skewness, and kurtosis. 

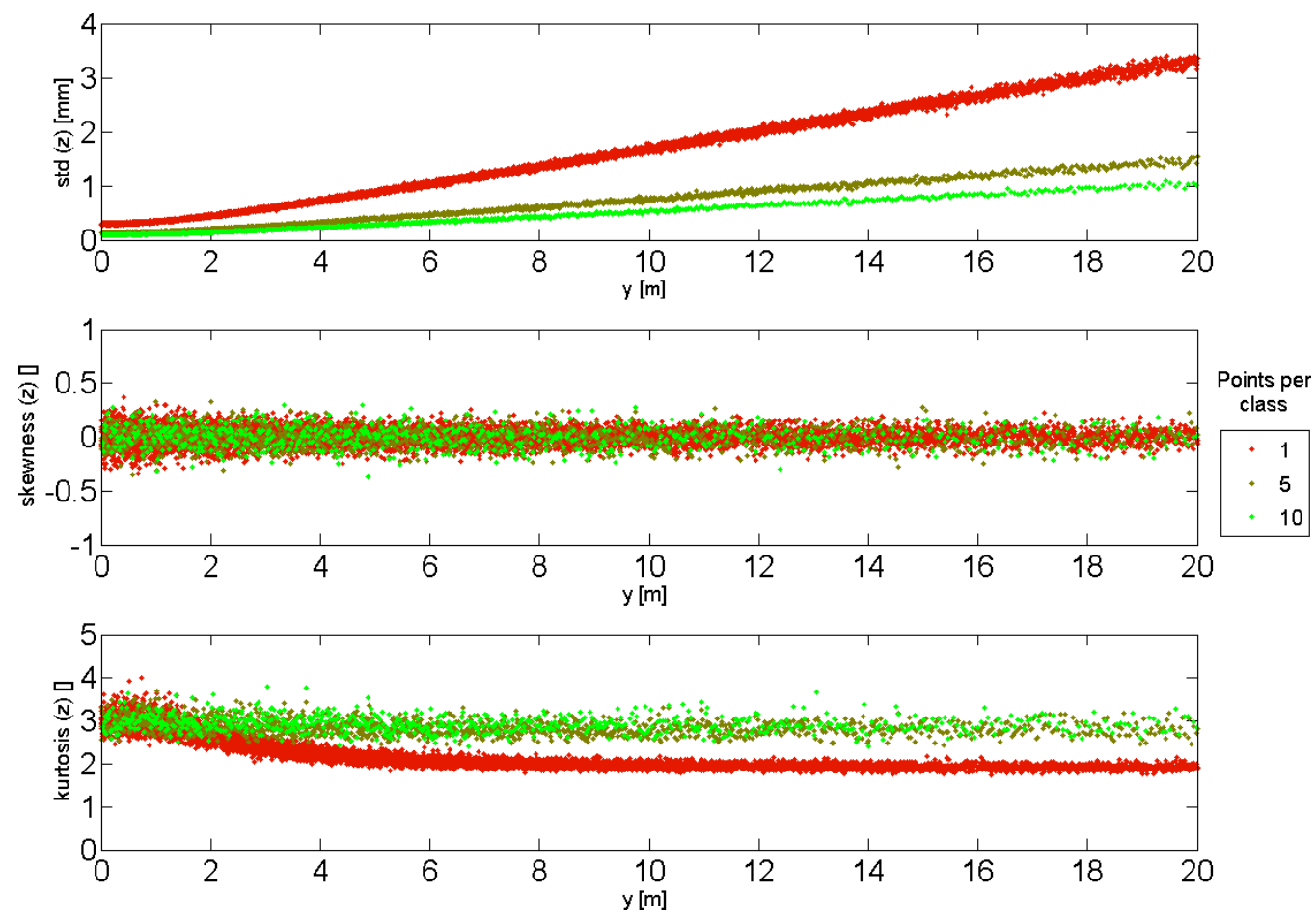

Figure 7. Real data: analysis of the observed k-TLS profiles for three different class widths: standard deviations, skewness, and kurtosis.

mitigated in case of wider classes. Both effects were also obtained in Simulation II shown in Figure 5 by modeling of a uniformly distributed uncertainty component for the angular increment of the vertical servo-motor. Note that the visible variability of the values decreases when $y$ increases.

Obviously, the real-data results fit quite well to the results of Simulation II which could be obtained using a rather basic uncertainty model with a few input parameters only. In addition to the simulations there are some further effects in the real data which could not be modeled up to now. Looking, e. g., at the subfigures of Figure 7 in total, some regions of horizontal distances $y$ can be identified where the values of the central moments are obviously disturbed. This holds in particular for the standard deviations like, e. g., between $16 \mathrm{~m}$ and $17 \mathrm{~m}$; there are also some periodic characteristics. A following study is required which aims at a refined statistical modeling and analysis of the k-TLS profile time series.

\section{CONCLUSIONS}

In this paper the 2D case of kinematic TLS was studied where repeated profile scans are observed from a fixed station with a high repetition frequency for monitoring purposes. The focus was put on a refined modeling of the uncertainty of both the observations and the derived positions of the profile points. In order to take into account the complete data processing chain, the strategy for generating and analyzing time series was considered. MC simulation techniques were applied to provide numerical results for discussion and validation. It turned out that a rather small number of input parameters for the uncertainty model are required to obtain simulation results which fit quite well to actually observed data. These real data were observed on the occasion of loading tests at an Autobahn bridge in southern Germany.

Further work has to address two main topics: the more refined simulation of more complex configurations by taking more parameters for the uncertainty model into account, and the rigorous and thorough statistical analysis of the real data in order to improve the physical observation models in case of k-TLS. The solution of both problems is essential for the highly sensitive and physically meaningful application of k-TLS techniques for monitoring of, e. g., large structures such as bridges.

\section{References}

[1] Alkhatib H., On Monte Carlo methods with applications to the current satellite gravity missions, $\mathrm{PhD}$ thesis, Institute for Geodesy and Geoinformation of University of Bonn, 2007.

[2] Alkhatib H., Neumann I. and Kutterer H., Uncertainty modeling of random and systematic errors by means of Monte Carlo and fuzzy techniques, Journal of Applied Geodesy 3 (2009), $67-81$. 
[3] Gentle J. E., Random Number Generation and Monte Carlo Methods, Wiley, Springer, $2^{\text {nd }}$ edition, 2003

[4] Gibbons J. D. and Chakraborti S., Nonparametric statistical inference, volume 198 of Statistics, Chapman \& Hall/CRC Press, Boca Raton and Fla, $5^{\text {th }}$ edition, 2011.

[5] Hennes M., Konkurrierende Genauigkeitsmasse: Potential und Schwäachen aus der Sicht des Anwenders, AVN 4 (2007), 136-146.

[6] ISO, Guide to the expression of uncertainty in measurements (GUM), International Organization for Standardization, Geneva, 1995.

[7] ISO, Evaluation of measurement data - Supplement 1 to the Guide to the expression of uncertainty in measurement - Propagation of distributions using a Monte Carlo method., Joint Committee for Guides in Metrology, Bureau International des Poids et Mesures, Geneva, 2007.

[8] Koch K. R., Introduction to Bayesian Statistics, Springer, Berlin - Heidelberg - New York, $2^{\text {nd }}$ edition, 2007.

[9] Koch K. R., Evaluation of uncertainties in measurements by Monte Carlo simulations with an application for laserscanning, J. Applied Geodesy 2 (2008), 67-77.

[10] Koch K. R., Determining uncertainties of correlated measurements by Monte Carlo simulations applied to laserscanning, J. Applied Geodesy 2 (2008), 139-147.
[11] Kutterer H., Alkhatib H., Paffenholz J.-A. and Vennegeerts H., Monte-Carlo simulation of profile scans from kinematic TLS, in: Proceedings of the FIG Congress 2010, http://http://www.?g.net/pub/?g2010/papers/ ts03d\%5Cts03dkuttereralkhatibetal3795.pdf.

[12] Martinez W. L. and Martinez A. R., Computational statistics handbook with MATLAB, volume 8 of Computer science and data analysis series, Chapman $\&$ Hall/CRC, Boca Raton, $2^{\text {nd }}$ edition, 2008.

[13] Reshetyuk Y., Investigation and calibration of pulsed time-of-flight terrestrial laser scanners, Licentiate thesis in Geodesy, Sweden, 2006.

[14] Schulz T. and Ingensand H., Infuencing Variables, Precision and Accuracy of Terrestrial Laser Scanners, in: Intergeo East, Bratislava, Slovakia, 2004.

[15] Siebert B. R. L. and Sommer K.-D., Weiterentwicklung des GUM und Monte-Carlo-Techniken, Technisches Messen 71 (2004), 67-80.

[16] Vennegeerts H., Liebig J. P., Hansen M., Neuner H., P., J.A., Grünberg J., and Kutterer H., Monitoring eines Brückentragwerks Vergleichende Messungen mit einem terrestrischen Laserscanner und Sensoren der Baumesstechnik, in: Wunderlich T., editor, Ingenieur-Vermessung 2010, Wichmann, Heidelberg, 2010. 
Bereitgestellt von | Technische Informationsbibliothek (TIB) Angemeldet 